\title{
Experimental investigation of quenching behavior of heated zircaloy rod in accidental condition of nuclear reactor with water and water based nanofluids
}

\author{
A. S. Chinchole, P. P. Kulkarni, A. K. Nayak \\ Reactor Engineering Division, Bhabha Atomic Research Centre, Trombay Mumbai 400085, India \\ chinchole@barc.gov.in, parimalk@barc.gov.in, arunths@barc.gov.in
}

PACS 87.85.Rs

DOI 10.17586/2220-8054-2016-7-3-528-533

\begin{abstract}
The physical phenomena of rewetting and quenching are of prime importance in nuclear reactor safety in the event of Loss of Coolant Accident (LOCA). In such a case, the fuel pins become dry hot. Under this condition, cold water is injected from emergency core cooling system (ECCS). The quenching behavior of such heated rod bundle (re-flood heat transfer behavior) is quite complex. It is well known that Nanofluids have better heat removal capability and a high heat transfer coefficient owing to their enhanced thermal properties. Recent investigations have shown that the addition of the $\mathrm{Al}_{2} \mathrm{O}_{3}$ nanoparticles result in better cooling capabilities as compared to the traditionally used quenching media. In this context, the authors have carried out experiments on quenching behavior of hot zircalloy tube with water and nanofluids as stated above. Quenching of the tube was observed to occur within few seconds in both the cases in the presence of decay heat. It was also observed that the nanofluids showed slightly reduced quenching time as compared to water.
\end{abstract}

Keywords: Rewetting, quenching, $\mathrm{Al}_{2} \mathrm{O}_{3}$ nano-fluids, zircaloy tube.

Received: 4 February 2016

Revised: 10 April 2016

\section{Introduction}

Quenching still remains one of the most widely used industrial heat treatment processes. This means the rapid cooling of high temperature materials mainly in demineralied (DM) water, oil and molten salt or polymer solution baths, which is frequently encountered in various engineering applications, such as heat treatments of steel, safety of nuclear reactors, and the rapid solidification processing. Thus, an acceleration of the transition from film boiling to nucleate boiling is often desirable, as it results in a much higher heat transfer rate. A new type of quenching media has recently been developed and today world's leading scientific institutions are conducting research to find out more about this medium called nanofluid. Nanofluids are colloidal suspensions of stably dispersed nanoparticles in base fluids. The term was first introduced by Das et al. [1] in 1995. Nanoparticles dispersed in base fluid can be metal oxides, such as $\mathrm{CuO}, \mathrm{Al}_{2} \mathrm{O}_{3}$ and $\mathrm{TiO}_{2}$ [2-5] and for base fluid, most commonly deionized or fresh water is used. Today there is no exact confirmed theoretical background available that would explain all nanofluid properties. Nevertheless, the use of nanofluids as a heat transfer medium has gained a lot of momentum. Nanofluids are of interest to a variety of industry branches due to a wide range of possible applications such as heat transfer applications, automotive, electronics and biomedical applications. At MIT [3] quenching of steel and zircaloy spheres in nanofluids with $0.1 \% \mathrm{Al}_{2} \mathrm{O}_{3}$ was studied. They reported that the main reason for quenching acceleration is the surface roughness increase and the wettability enhancement due to nanoparticle deposition. They observed a thin layer of nanoparticles on sphere surface. Therefore, the deposition of nanoparticles prevented the formation of vapor film and resulted in quick quenching of the hot sphere.

Buongiorno and $\mathrm{Hu}$ [5] studied the boiling behavior of nanofluids, prominently CHF limit and quenching phenomena. The nanoparticle deposition layer was found to increase the surface roughness and wettability, both of which could be linked to CHF enhancement and quenching acceleration. Kim et al. [3] observed that nanoparticles dispersed in the fluid at low concentration $(<0.1 \%)$ did not change the quenching process. Nanoparticles deposited on the rodlet surface are responsible for accelerated quenching. The quenching acceleration strongly depends on the material used for nanoparticle. Ciloglu and Bolukbasi [6] investigated the quenching behavior of aqueous nanofluids containing various volume fractions of $\mathrm{Al}_{2} \mathrm{O}_{3}, \mathrm{SiO}_{2}, \mathrm{TiO}_{2}$ and $\mathrm{CuO}$ nanoparticles around high temperature brass rod experimentally. They observed that after the repetition tests in nanofluids, a nanoparticle porous layer occurs on the quenched surface and thus the film boiling vanishes. The results show that contact angle decreases and surface roughness increases. They concluded that the primary reason of CHF enhancement is the change of the surface characteristics due to the porous layer. Application of nanofluids as quenching media in quenching process was also tested experimentally in 2004 by Das et al. [7]. High temperature copper spheres were quenched in alumina nanofluids in order to investigate the effect suspended nanoparticles on film boiling heat 
transfer characteristic. Even though high particle concentrations were used, from 5 to 20 vol.\%, nanofluids showed lower boiling heat transfer rate than pure DM water. More important, the experiments conducted on a previously quenched sphere resulted in a much more rapid process compared to quenching of a clean sphere.

Whereas most of the work has been cited on spheres, much less work has been carried out on rods, that too generating decay heat which is of a concern from nuclear safety. In this work, the authors have carried out experiments on the quenching behavior of hot zircalloy tube with DM water and metal oxide nanofluids as above. Tube quenching was observed within a few seconds, even with the presence of decay heat.

\subsection{Quenching process}

In the quenching process, the hot components are cooled down to room temperature by immersion into a liquid, spraying with a liquid or by pouring over a liquid (i.e. quench media). The quenching medium can be DM water, oils and aqueous polymer solutions, nanofluids etc. Before a component is quenched, it is heated to a uniform temperature and then is rapidly cooled by the cooling medium. During this process, thermal stresses are developed due to a high temperature gradient in the material. Residual stresses remaining in the material are undesirable because they may cause unpredictable distortions and have a deleterious effect on fracture and corrosion performance.

The quenching process is a complex thermodynamic and fluid dynamic problem consisting of several different phases. If the quenching media is vaporizable liquid, four modes of cooling can be observed: i) shock film boiling, ii) full film boiling, iii) nucleate boiling and iv) convection cooling mode (Fig. 1). Each of these modes is characterized by different surface heat transfer coefficients (HTC) and cooling rates.

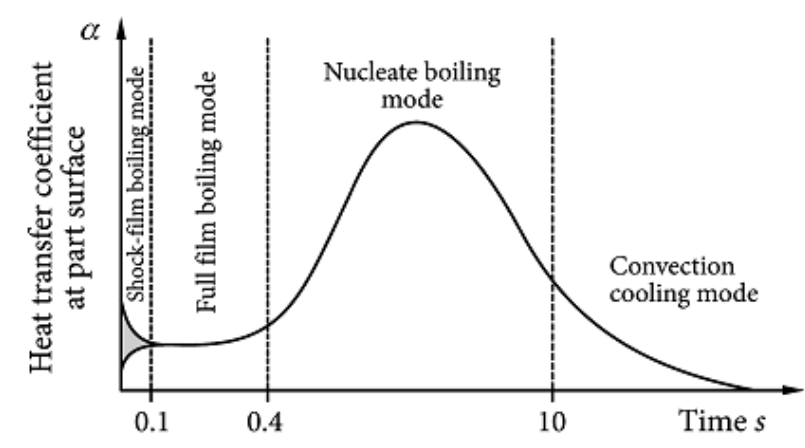

FIG. 1. Different types of cooling at quenching process

\subsection{Experiment on quenching with hot zircalloy rod}

This system is designed to study the clad performance during Emergency Core Cooling System (ECCS) activation in a nuclear reactor during Loss of Coolant Accident (LOCA) scenario when cold DM water is injected over hot clad surface. During ECCS injection, DM water is sprayed onto the hot rod in the form of a jet only from front direction. The objectives of these experiments are to determine the extent of quenching with presence of decay heat, study the radial temperature variation in the tube and study the extent of enhancement on quenching by use of nanofluids.

\section{Experiments conducted}

\subsection{Experimental setup}

The experimental setup is as shown in Fig. 2. Two concentric stainless steel pipes 50 NB (Sch. 30) and 40 NB (Sch. 30) form the vessel. The inner pipe has been perforated, with $1.5 \mathrm{~mm}$ diameter holes along the length having $10 \mathrm{~mm}$ linear pitch and 450 angular pitch, to allow DM water and nanofluids to inject over the tube surface. The hollow zircalloy tube (16 OD, $0.5 \mathrm{~mm}$ thick) is placed at the center of the vessel. The gap between inner perforated pipe and zircalloy tube is $12.9 \mathrm{~mm}$. A small uniform gap of $2.86 \mathrm{~mm}$ has been maintained in the annular space so that DM water and nanofluids jets attain sufficient velocity to reach hot zircalloy tube surface. Both pipes are welded to two $150 \mathrm{lbs}$ blind flange at either ends. Two nickel plugs are used to connect the zircalloy tube to power supply for direct resistance heating. Two central 'through' hole have been provided on the Nickel plug to vent out air from the Zircalloy tube which expands due to heating. Vent holes of $5 \mathrm{~mm}$ diameter (4 Nos.) are provided on the top flange to release steam along with hydrogen generated (if any) and air during the experiment. 
DM water and nanofluids will be supplied from radial direction with four inlet pipes (10 NB) on the outer pipe in to the annular space at a location near top of the set up and the same will be collected in to the drain at the bottom after impinging over hot zircalloy tube surface. The whole setup will be supported at a distance of $45 \mathrm{~mm}$ from the ground. The tube was equipped with $\mathrm{K}$ type SS sheathed thermocouple of $1.5 \mathrm{~mm}$ diameter brazed at different axial and locations along the length at uniform spacing of $30 \mathrm{~mm}$. Thermocouples are also brazed on the diagonally opposite location on the surface behind the spray nozzle location. Tube Surface temperature was measured over the total heated length. All these measurements will be recorded by a portable recorder at a span of $125 \mathrm{~ms}$.

Figure 3 shows the location of thermocouple position on the zircaloy tube in the experimental setup. Each thermocouple was situated within a specific location on surface of the zircaloy tube.

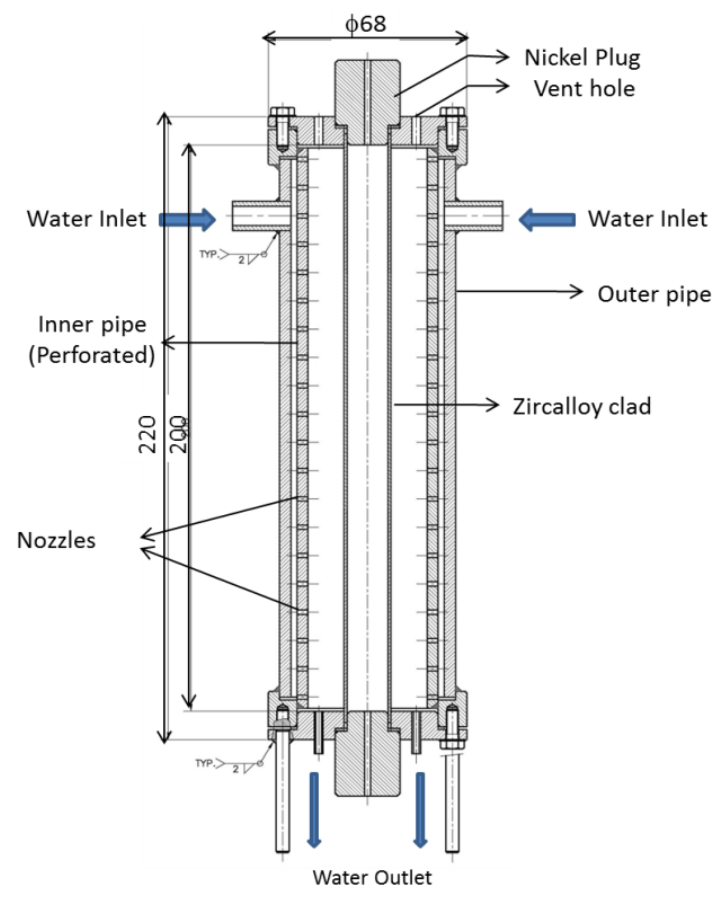

FIG. 2. Experimental setup

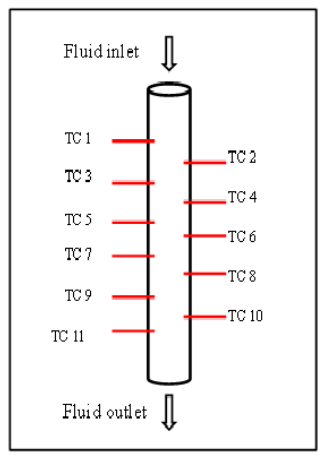

FIG. 3. Thermocouple position on zircaloy tube

\subsection{Experimental procedure for nanofluids}

In the present experiment, the zircalloy tube was heated gradually in a stepwise manner from ambient temperature to a maximum temperature of $650{ }^{\circ} \mathrm{C}$. The tube was maintained at the required temperature for 3 minutes and then DM water was injected through a flexible inlet pipe using a pump with a constant flow rate of $10 \mathrm{lpm}$. Initially, the experiment was carried out with $\mathrm{DM}$ water. The experiment was repeated with $\mathrm{Al}_{2} \mathrm{O}_{3}$ nanofluid of different concentrations to check the quenching phenomena for zircaloy tube with $\mathrm{Al}_{2} \mathrm{O}_{3}$ nanofluids.

\subsection{Preparation and characterization of nanofluids}

An aqueous solution of nanofluids was prepared by adding desired concentration (by weight) of $\mathrm{Al}_{2} \mathrm{O}_{3}$ (Alumina) nanopowder of particle size $10-100$ nanometer and $99.7 \%$ purity to the DM water. The reason for using $\mathrm{Al}_{2} \mathrm{O}_{3}$ nanoparticle is the fact that the thermo-physical characteristics of the base fluid (DM water) is most widely known and the thermal properties of $\mathrm{DM}$ water $-\mathrm{Al}_{2} \mathrm{O}_{3}$ nanofluids for different particle concentration have already been studied [7]. To prevent the particles from agglomerating and settling, the suspension was sonicated in an ultrasonic bath. The dispersion of the particles was first done by mixing the required volume of powder in a chemical measuring flask with DM water and then using ultrasonication with frequency $50 \mathrm{~Hz}$ and power of $120 \mathrm{~W}$. After making a proper mixture, the flask was kept again under ultrasonic treatment for about $4 \mathrm{~h}$, which is a sufficient time to ensure stable particle dispersion in DM water without agglomeration [8]. The size of nanoparticles in powder form was characterized by transmission electron microscope (TEM). After preparing the 
nanofluid, it was poured in the storage tank of the apparatus and the experiment was repeated for nanofluids with different concentrations.

The nano fluid is used immediately after its preparation so as to avoid any issue regarding its stability. Since the wt. \% of nanoparticle used in the preparation of nanofluids is very small, the agglomeration begins only after 24 hours of its preparation.

\section{Results and discussion}

The following are the Transmission Electron Microscopic images of $\mathrm{Al}_{2} \mathrm{O}_{3}$ nanoparticles before and after the quenching process.

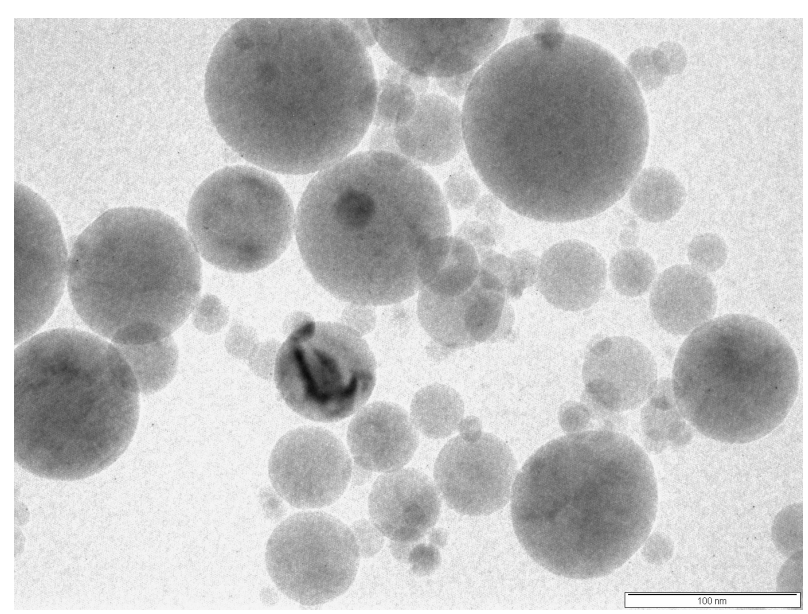

(a)

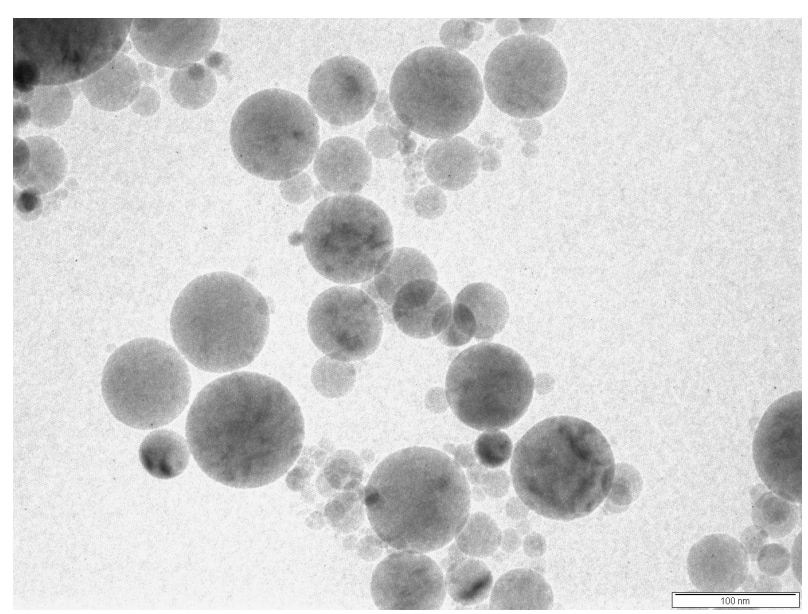

(b)

FIG. 4. TEM images of $\mathrm{Al}_{2} \mathrm{O}_{3}$ Nanofluids (a) before Injection, (b) after injection

Figure 4 shows that TEM images, having resolution of $100 \mathrm{~nm}$, reveal that there is no change in the size of $\mathrm{Al}_{2} \mathrm{O}_{3}$ nano particles before and after their injection in the test apparatus for the quenching experiment.

Figure 5 shows the quenching data with DM water. All thermocouples showed rapid quenching from about $650{ }^{\circ} \mathrm{C}$ to DM water ambient temperature within $15 \mathrm{~s}$ period. There is slight delay in onset of quenching of thermocouples TC7 and TC11. This is due to the fact that, they are located diametrically opposite on the tube from the entry of DM water. However, complete quenching is eventually observed.

Figure 6 shows the quenching data with 0.01 wt.\% $\mathrm{Al}_{2} \mathrm{O}_{3}$ nanofluids. All thermocouples showed rapid quenching from about $650{ }^{\circ} \mathrm{C}$ to $\mathrm{DM}$ water ambient temperature within a $15 \mathrm{~s}$ period. There is slight delay in

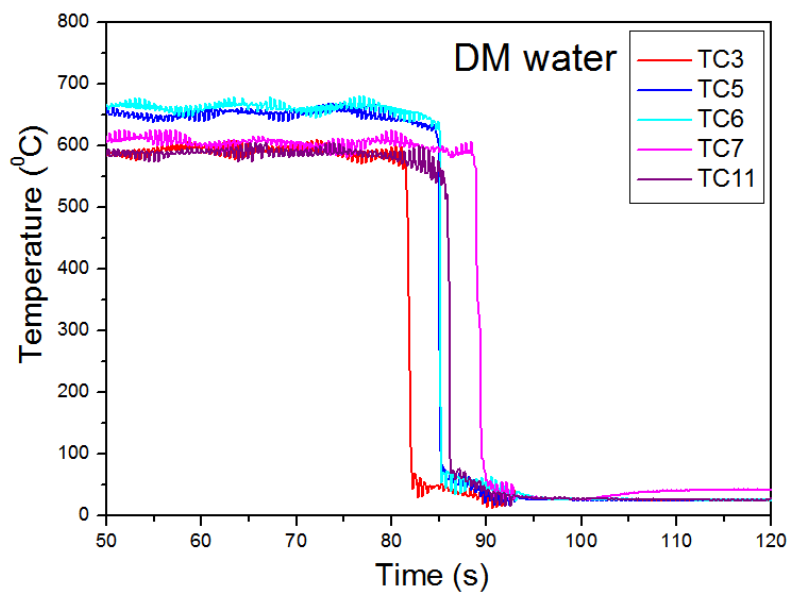

FIG. 5. Quenching time for DM DM water

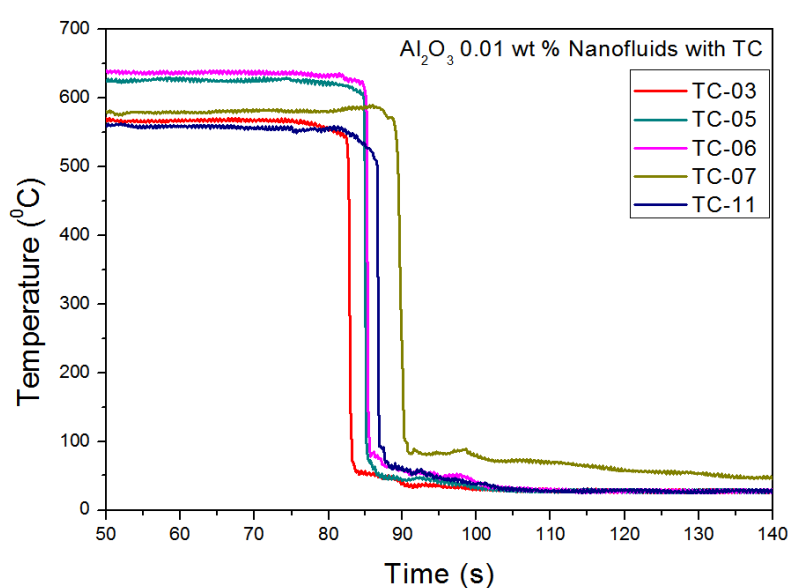

FIG. 6. Quenching time for 0.01 wt.\% $\mathrm{Al}_{2} \mathrm{O}_{3}$ Nanofluids on all TC 
onset for the quenching of thermocouples TC7 and TC11. This is due to the fact that, they are located diametrically opposite on the tube from the entry of DM water. However, complete quenching is eventually observed.

Figure 7 shows the quenching data with $\mathrm{DM}$ water $\& \mathrm{Al}_{2} \mathrm{O}_{3}$ nanofluids with different concentrations at location TC-1. Since TC-1 is near the top surface, there is heat loss which reduces the maximum temperature to about $650{ }^{\circ} \mathrm{C}$. It is seen that rapid quenching from about $650{ }^{\circ} \mathrm{C}$ to water ambient temperature within $15 \mathrm{~s}$ period. Complete quenching was observed for different concentrations of nanofluids. Different concentrations of nanofluids showed different rates of quenching, based on the particles' concentration as compared to DM water. Figure shows that the zircaloy tube became quenched slightly faster by using nanofluids as compared to the DM water. All the temperature profiles depended upon the thermocouple position of the zircaloy tube.

Figure 8 shows the temperatures on the tube at axially different positions. TC 11, which is at the bottom, was shown to be quenched very quickly due to the fact that the DM water is accumulated at the bottom first. TC 7, which is opposite in the middle of the tube, takes almost an additional $10 \mathrm{~s}$ for quenching which is the delay that caused the slight rise in temperature. Nevertheless, there is no increase in temperature and complete quenching is observed.

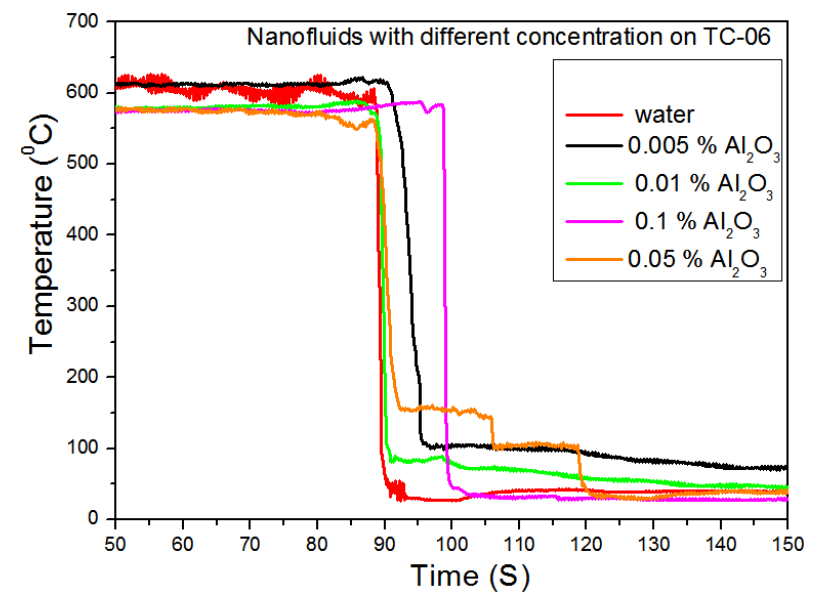

FIG. 7. Quenching time for $\mathrm{Al}_{2} \mathrm{O}_{3}$ nanofluids with different concentration on TC 6

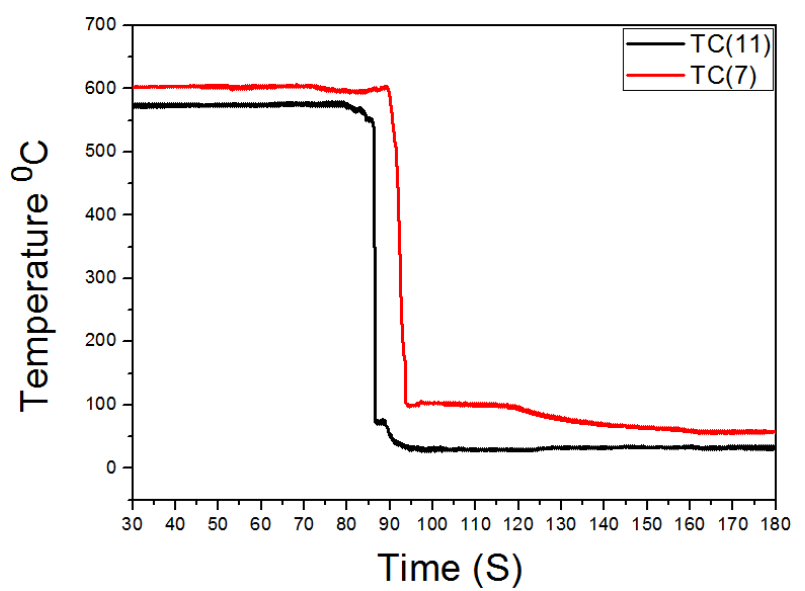

FIG. 8. Comparison between front \& backside thermocouple

Figure 9 shows the difference in onset of quenching because of location. The thermocouple located at the diametrically opposite position shows quenching after delay of about $30 \mathrm{~s}$. This is due to the fact that, when the DM water is sprayed from front, the thermocouple located there shows immediate quenching. However, the backside thermocouple quenches only when the sprayed DM water falls down and fills the entire cavity which introduces certain delay. But nevertheless, there is complete quenching of the tube.

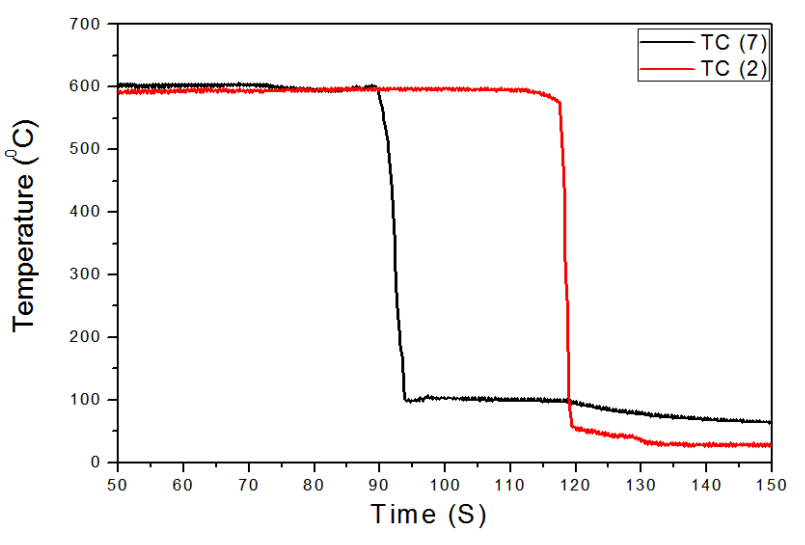

FIG. 9. Frontside \& backside thermocouple in experimental facility

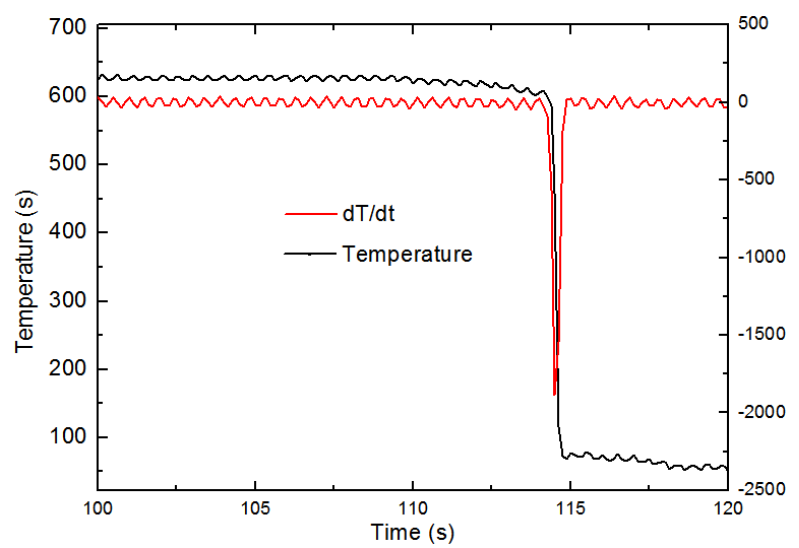

FIG. 10. Quenching time for $\mathrm{Al}_{2} \mathrm{O}_{3}$ nanofluid 
In order to measure the exact quenching time, the rate of change of temperature was plotted as shown in Fig. 10. There, a sharp change in the slope can be seen for the temperature at the onset and completion of quenching. The difference between these two readings gives an accurate measure of quenching time.

Quenching times varied from $0.6 \mathrm{~s}$ to $0.9 \mathrm{~s}$ for all the fluids, as shown in Fig. 11. The quenching times for nanofluids were shown to be marginally reduced. It is seen that at lower concentrations, the quenching times are less, however at higher concentrations, there is a slight increase in the quenching time. The reason can be attributed to formation of a nanolayer on the rod. A similar process occurs during boiling in nanofluid [9]. At lower concentrations, there is a fine layer formation, which assists the wetting of the rod and thus reduces the quenching time. At higher concentrations, there is a thick nanolayer formation, which changes the wetting characteristics of the rod which in turn delays the quenching. Nevertheless, the thick nanolayer has better wetting characteristics than the bare rod.

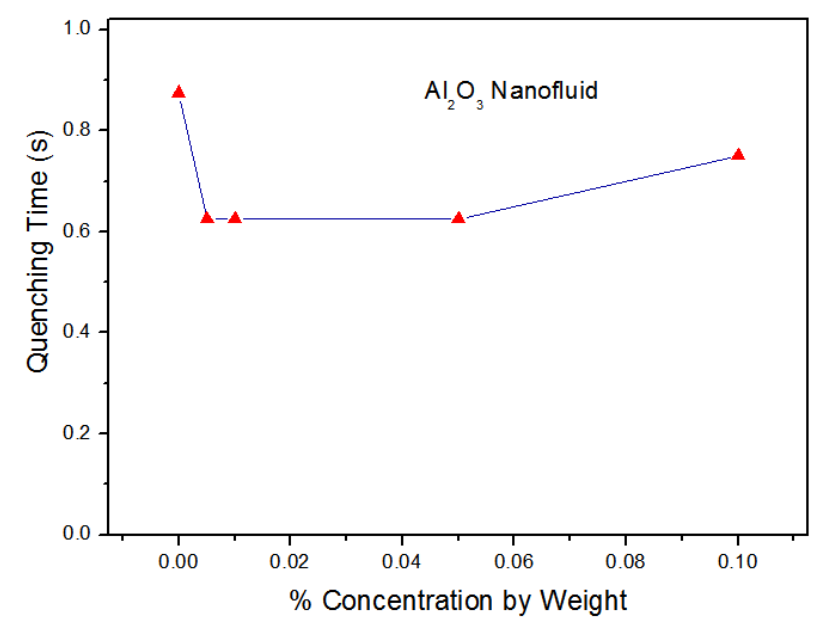

FIG. 11. Quenching time for $\mathrm{Al}_{2} \mathrm{O}_{3}$ nanofluid

\section{Conclusions}

In this work, quenching behavior of hot zircalloy tube was studied. It was observed that, even with the presence of decay heat, the tube was quenched within a short period of time. Quenching behavior was shown to be more or less similar for all the fluids, however, nanofluids did exhibit slightly reduced quenching times.

\section{References}

[1] Das S.K., Choi S.U.S., Yu W., Pradeep T. Nanofluids: Science and Technology. John Wiley \& Sons, Inc., Hoboken, New Jersey, 2008.

[2] Kim H., Kim J., Kim M. Experimental study on CHF characteristics of $\mathrm{DM}_{\text {water-TiO }}$ nano-fluids. Nuclear engineering and technology, 2006, 38 (1), P. 61-68.

[3] Kim H., DeWitt G., et al. On the quenching of steel and zircaloy spheres in DM water-based nanofluids with alumina, silica and diamond nanoparticles. International Journal of Multiphase Flow, 2009, 35, P. 427-438.

[4] Kim H., Buongiorno J., Hu L., McKrell T. Nanoparticle deposition effects on the minimum heat flux point and quench front speed during quenching in DM water-based alumina nanofluids. International Journal of Heat and Mass Transfer, 2010, 53, P. 1542-1553.

[5] Buongiorno J. et al. A benchmark study on the thermal conductivity of nanofluids. Journal of Applied Physics, 2009 , 106, P. 094312 (14 p.).

[6] Ciloglu D., Bolukbasi A., Comakli K. Effect of Nanofluids on the Saturated Pool Film Boiling. World Academy of Science, Engineering and Technology, 2012, 6, P. 7-29.

[7] Park H.S., Shiferaw D.,et al. Film boiling heat transfer on a high temperature sphere in nanofluid. Proceedings of 2004 ASME Heat Transfer/Fluids Engineering Summer Conference, 2004, P. 1-8NC.

[8] Das S.K., Putra N., Thiesen P., Roetzel W. Temperature dependence of thermal conductivity enhancement for nanofluids. Journal of Heat Transfer - Transactions of the ASME, 2003, 125, P. 567-574.

[9] Nayak A.K., Kulkarni P.P., Chinchole A.S. Experimental Investigation on Pool Boiling Critical Heat Flux with Nanofluids. Journal of Nanofluids, 2015, 4, P. 140-146. 